\title{
Effects of antibodies against human parvovirus B19 on angiogenic signaling
}

\author{
CHIA-CHENG SU ${ }^{1}$, TSAI-CHING HSU ${ }^{2-4}$, CHAO-HSIANG HSIAO ${ }^{2}$, \\ CHUN-CHING CHIU ${ }^{2,5}$ and BOR-SHOW TZANG ${ }^{2-4,6}$ \\ ${ }^{1}$ Department of Urology, Chi Mei Medical Center, Tainan 710; ${ }^{2}$ Institute of Biochemistry, Microbiology \\ and Immunology, College of Medicine; ${ }^{3}$ Immunology Research Center, Research and Development, \\ Chung Shan Medical University; ${ }^{4}$ Department of Clinical Laboratory, Chung Shan Medical \\ University Hospital, Taichung 402; ${ }^{5}$ Department of Neurology and Department of Medical \\ Intensive Care Unit, Changhua Christian Hospital, Changhua 500; ${ }^{6}$ Department of Biochemistry, \\ School of Medicine, Chung Shan Medical University, Taichung 402, Taiwan, R.O.C.
}

Received June 7, 2019; Accepted November 18, 2019

DOI: $10.3892 / \mathrm{mmr} .2020 .10921$

\begin{abstract}
Human parvovirus B19 (B19V) infection has symptoms similar to those of anti-phospholipid syndrome (APS). Antibodies against B19V-VP1 unique region (VP1u) exhibit activity similar to that of anti-phospholipid antibodies (aPLs) by inducing vascular endothelial cell adhesion factors and APS-like syndrome. Previous studies have identified an effect of aPLs on angiogenesis. However, little is understood regarding the effect of anti-B19V-VP1u antibodies on angiogenesis. The present study investigated the effects of anti-B19V-VP1u antibodies on the expression of adhesion molecules and angiogenic signaling using an aPL-induced human umbilical vein endothelial cell (HUVEC) model, and trypan blue staining and western blotting. The effect of B19V-VP1u antibodies on vascular endothelial growth factor (VEGF) expression in A549 cells, another well-known model used to study angiogenesis, was also examined. Significantly higher intracellular adhesion molecule 1 expression was observed following treatments with $10 \%$ fetal calf serum (FCS), aPL immunoglobulin G (IgG), B19V-VP1u IgG or B19V-NS1 IgG, compared with in the normal human (NH) IgG-treated cells. Conversely, significantly higher vascular cellular adhesion molecule 1
\end{abstract}

Correspondence to: Professor Bor-Show Tzang, Department of Biochemistry, School of Medicine, Chung Shan Medical University, 110 Section 1, Jianguo North Road, Taichung 402, Taiwan, R.O.C.

E-mail: bstzang@csmu.edu.tw

Dr Chun-Ching Chiu, Department of Neurology and Department of Medical Intensive Care Unit, Changhua Christian Hospital, 135 Nanxiao Street, Changhua 500, Taiwan, R.O.C.

E-mail: s31065@gmail.com

Key words: human parvovirus B19, VP1 unique region, anti-phospholipid antibodies, human umbilical vein endothelial cells, angiogenesis was only detected in HUVECs treated with B19V-VP1u IgG. Significantly increased integrin $\beta 1$ was detected in HUVECs treated with aPL IgG or B19V-VP1u IgG, whereas no difference in integrin $\beta 1$ was observed in those treated with $10 \%$ FCS, NH IgG or B19V-NS1 IgG. No difference in AKT-mTOR-S6 ribosomal protein (S6RP) signaling was observed in HUVECs treated with B19-VP1u IgG or B19V-NS1 IgG, compared with NH IgG-treated cells. Significantly higher human inducible factor-1 $\alpha$ was detected in HUVECs treated with $10 \%$ FCS, aPL IgG, B19V-VP1u IgG or B19V-NS1 IgG, compared with in $\mathrm{NH} \mathrm{IgG-treated} \mathrm{cells.} \mathrm{However,} \mathrm{there} \mathrm{was} \mathrm{no} \mathrm{difference} \mathrm{in}$ the level of VEGF observed among HUVECs treated with $\mathrm{NH}$ IgG, B19V-VP1u IgG or B19V-NS1 IgG. Notably, no difference in VEGF level was observed in A549 cells treated with $\mathrm{NH}$ IgG, aPL IgG, B19V-VP1u IgG or B19V-NS1 IgG. These findings suggest that anti-B19V-VP1u antibodies may serve a role in activating adhesion molecules, but not in AKT-mTOR-S6RP signaling.

\section{Introduction}

Human parvovirus B19 (B19V) is a member of the Parvoviridae family and has been demonstrated to cause various human diseases, including erythema infectiosum, arthropathies, hemolytic disorders, hydrops fetalis and fetal death (1-4). Although the role of $\mathrm{B} 19 \mathrm{~V}$ in inducing autoimmunity is still unknown, numerous studies have reported an association between B19V infection and autoimmune disorders, such as systemic lupus erythematous (SLE) and rheumatoid arthritis (RA) $(5,6)$. The VP1 unique region (VP1u) of B19V exhibits activity of secreted phospholipidase A2 and has been demonstrated to have an essential role in viral infectivity, and the induction of inflammation and autoimmunity (5,7-10). Indeed, evidence indicates a link between B19V and autoimmune disorders, particularly an association between the B19V-VP1u and anti-phospholipid syndrome (APS) $(8,10-12)$.

APS is an autoimmune state characterized by thrombosis, which affects the venous or arterial vascular systems, and 
obstetrical morbidity (13). APS is associated with numerous autoimmune diseases, including SLE, systemic sclerosis, Sjögren's syndrome, dermatomyositis and RA $(13,14)$. APS is considered to be an autoantibody-mediated disease diagnosed by the presence of anti-phospholipid antibodies (aPLs), such as anti-cardiolipin and anti- $\beta$-2-glycoprotein I ( $\beta 2 \mathrm{GPI})$ antibodies (13). A number of studies have reported an association between B19V infection and the induction of APL $(12,15)$. The induction of APS-like syndromes and aPLs has been observed in naïve mice that received antibodies against B19V-VP1u (8). Notably, similar activity between APL and B19V-VP1u antibodies on the activation of adhesion molecules has been reported (16). Our previous study demonstrated that B19V-VP1u antibodies induce the expression of adhesion molecules, including intracellular cell adhesion molecule 1 (ICAM-1), vascular cell adhesion molecule 1 (VCAM-1) and E-selectin, in human vascular endothelial cells by activating the phosphorylated (p)-p38 mitogen-activated protein kinase (MAPK) signaling pathway (17). Since similar results were also reported in previous studies $(18,19)$, these findings indicated that both aPL and B19V-VP1u antibodies induce the expression of adhesion molecules through the p38 MAPK pathway.

Endothelial dysfunction is an important pathological process that is associated with APS. Evidence indicates that aPLs, including $\beta 2$ GPI antibodies, significantly reduce the length of the tubules that are formed in an in vitro endometrial endothelial cell angiogenesis assay and reduce vessel formation in an aPL-inoculated animal (20). In the presence of monoclonal aPLs or immunoglobulin Gs ( $\mathrm{IgGs}$ ) that have been isolated from patients with APS, impairments in vascular remodeling and angiogenesis have also been reported (21). In contrast, $\beta 2$ GPI antibodies that have been isolated from patients with APS induce angiogenesis by activating the mTOR pathway in cultured vascular endothelial cells (22). Although antibodies against B19V-VP1u have been demonstrated to induce APS-like symptoms in naïve mice, the effect of B19V-VP1u antibodies on angiogenesis remains unclear. The present study investigated the effect of antibodies against B19V-VP1u on mTOR signaling to identify the role of B19V-VPlu in angiogenesis in human umbilical vein endothelial cells (HUVEC) and the expression of vascular endothelial growth factor (VEGF) protein in A549 cells, another well-known model for angiogenesis study $(23,24)$.

\section{Materials and methods}

Antibody preparations. Human parvovirus B19V-NS1 and B19V-VP1u recombinant proteins, and antibodies against B19V-NS1 and B19V-VP1u were prepared as described in our previous studies $(17,25)$. The yields of the purified recombinant B19-NS1 and B19-VP1u proteins were 3.8 and $2.3 \mu \mathrm{g} / \mathrm{ml}$, respectively, with purities of $\sim 96.2$ and $\sim 98.3 \%$, respectively. The antibodies against B19V-NS1 and B19V-VP1u were purified by protein $\mathrm{G}$ agarose (Roche Diagnostics) chromatography. The purified antibodies were filtered through a 0.22- $\mu \mathrm{m}$ microporous membrane (EMD Millipore). Normal human serum IgG (NH IgG; $200 \mu \mathrm{g} / \mathrm{ml})$ and human aPL IgG $(200 \mu \mathrm{g} / \mathrm{ml} ; 250$ units) were obtained from a commercial APhL ELISA IgG and IgM horseradish peroxidase (HRP) kit (cat. nos. LAPL-K-HRP-00GM and LAPL-K-HRP-01GM; Louisville APL Diagnostics, Inc.). A limulus amebocyte lysate endochrome assay (Charles River Laboratories, Inc.) was used to detect the endotoxin levels of the antibody preparations. The endotoxin levels were identified to be below the detection limit ( 0.25 endotoxin $\mathrm{U} / \mathrm{ml}$ ) for all $\mathrm{IgG}$ preparations at the concentrations used in the present study.

Cell culture and treatment. HUVECs (BCRC no. H-UV001; cat. no. 01193) and a non-small cell lung cancer cell line (A549) were purchased from Bioresource Collection and Research Center, Food Industry Research and Development Institute. The culture dishes were pre-coated with $1 \%$ gelatin and HUVECs were then cultured in 90\% M199 medium (Invitrogen; Thermo Fisher Scientific, Inc.) containing $25 \mathrm{U} / \mathrm{ml}$ heparin, $30 \mu \mathrm{g} / \mathrm{ml}$ endothelial cell growth supplement, $1.5 \mathrm{~g} / \mathrm{l}$ sodium bicarbonate, 10\% FBS (Gibco; Thermo Fisher Scientific, Inc.) and $100 \mathrm{U} / \mathrm{ml}$ penicillin/streptomycin. A549 cells were cultured in DMEM (Invitrogen; Thermo Fisher Scientific, Inc.) containing 10\% FBS (Gibco; Thermo Fisher Scientific, Inc.) and $100 \mathrm{U} / \mathrm{ml}$ penicillin/streptomycin at $37^{\circ} \mathrm{C}$ in a $5 \% \mathrm{CO}_{2}$ incubator. The activation of p-AKT-mTOR-S6 ribosomal protein (S6RP) angiogenic signaling was performed as described previously (22). HUVECs were cultured in M199 medium containing 2\% fetal calf serum (FCS; Gibco; Thermo Fisher Scientific, Inc.) for $12 \mathrm{~h}$ at $37^{\circ} \mathrm{C}$ and subsequently treated with $5 \mu \mathrm{g} / \mathrm{ml} \beta 2 \mathrm{GP} 1$ (cat. no. OPPA01429; Aviva Systems Biology, Corp.) for $1 \mathrm{~h}$ at $37^{\circ} \mathrm{C}$. After washing with $1 \mathrm{X}$ PBS, the cells were cultured in M199 medium containing 2\% FCS and treated with $10 \%$ FCS or $100 \mu \mathrm{g} / \mathrm{ml} \mathrm{NH} \mathrm{IgG,} \mathrm{aPL} \mathrm{IgG,}$ B19V-VP1u IgG or B19V-NS1 IgG for a further 5 min at $37^{\circ} \mathrm{C}$. The reactions were then stopped by washing with ice-cold PBS and solubilizing in RIPA buffer (cat. no. 89901; Thermo Fisher Scientific, Inc.). The treatments with $10 \%$ FCS or NH IgG were considered as the positive control and negative control, respectively.

Viability assay. For cell survival determination, the Trypan blue exclusion method was conducted. Briefly, $5 \times 10^{5}$ HUVECs/well were cultured in a 6-well plate overnight at $37^{\circ} \mathrm{C}$ in an incubator. After $24 \mathrm{~h}, \mathrm{HUVECs}$ were cultured in M199 medium containing $2 \% \mathrm{FCS}$ for $12 \mathrm{~h}$ at $37^{\circ} \mathrm{C}$ and subsequently treated with $5 \mu \mathrm{g} / \mathrm{ml} \beta 2 \mathrm{GP} 1$ for $1 \mathrm{~h}$ at room temperature. After washing with $1 \mathrm{X}$ PBS, the cells were then cultured in M199 medium containing 2\% FCS and treated with $10 \%$ FCS or $100 \mu \mathrm{g} / \mathrm{ml} \mathrm{NH} \mathrm{IgG,} \mathrm{aPL} \mathrm{IgG,} \mathrm{B19V-VP1u}$ $\mathrm{IgG}$ or B19V-NS1 IgG for a further $10 \mathrm{~min}$ at $37^{\circ} \mathrm{C}$. Following the experimental treatments, the morphology of cells was observed and photographed under a phase-contrast microscope (Zeiss Axiovert 200; magnification, x200). Next, the culture medium was discarded, and the cells were harvested with $0.25 \%$ trypsin-EDTA solution. The number of viable cells was counted using Trypan blue solution for 1-2 min at room temperature under a Zeiss Axiovert 200 light microscope (magnification, $\mathrm{x} 200$ ). The viability of the control (untreated cells) was set as $100 \%$.

Protein preparation and immunoblotting. The cells were collected by centrifugation at $800 \mathrm{x}$ g for $5 \mathrm{~min}$ at $4^{\circ} \mathrm{C}$ and suspended in $600 \mu \mathrm{l}$ PRO-PREP ${ }^{\mathrm{TM}}$ buffer (iNtRON 
A
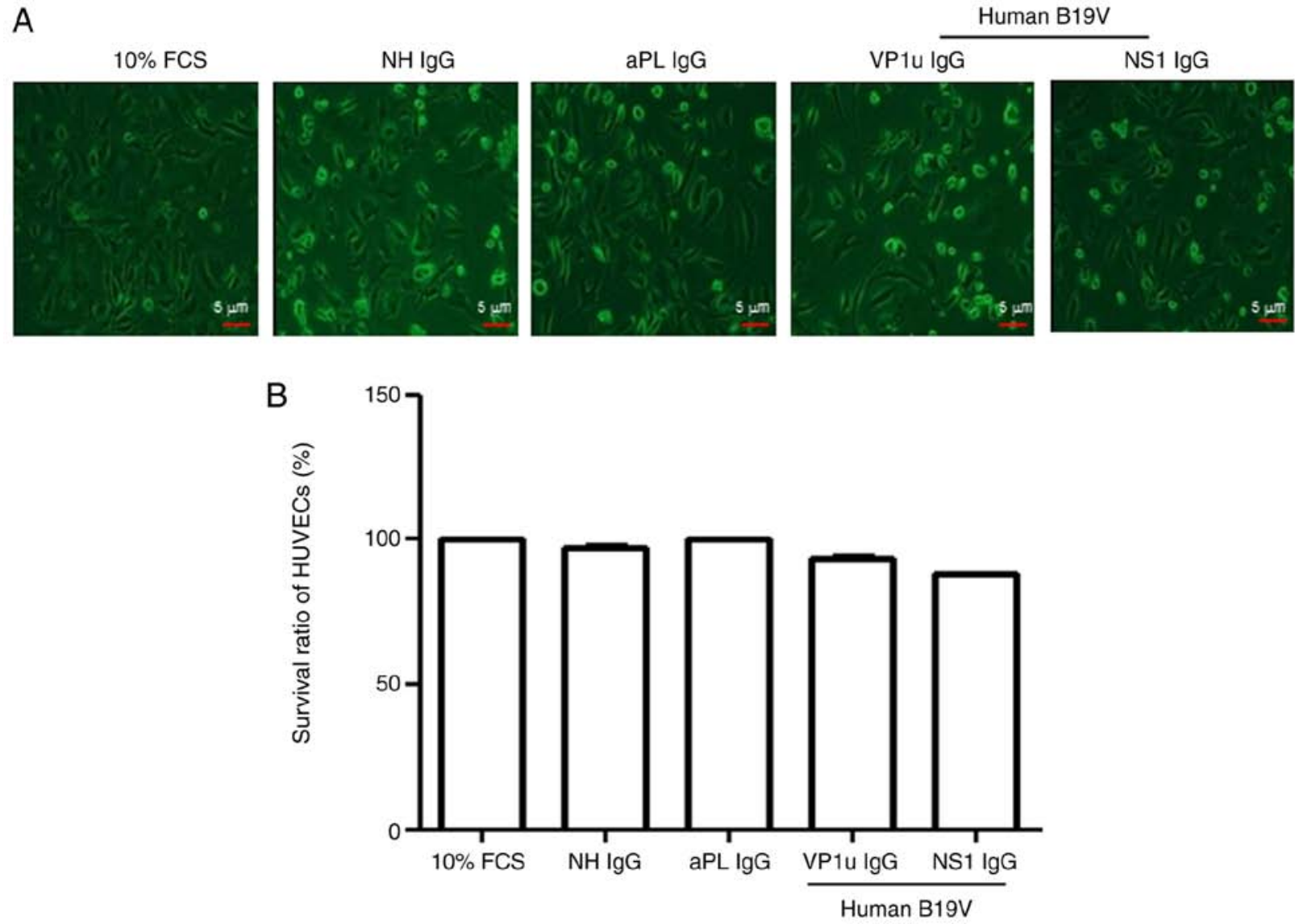

Figure 1. Viability of HUVECs. (A) Representative photographs of HUVECs in the presence of different antibodies for 10 min observed under a phase-contrast microscope (Zeiss Axiovert 200; magnification, x200). (B) Survival ratio of HUVECs in the presence of different antibodies for 10 min. Each experiment was repeated at least three times. HUVEC, human umbilical vein endothelial cell; IgG, immunoglobulin G; NH, normal human; FCS, fetal calf serum; aPL, anti-phospholipid antibodies; B19V, parvovirus B19; VP1u, VP1 unique region.

Biotechnology,Inc.) for lysis. The supernatant of protein extracts was then collected by centrifugation at $16,600 \mathrm{x} \mathrm{g}$ for $5 \mathrm{~min}$ at $4^{\circ} \mathrm{C}$. Protein concentration was determined by a modified Bradford's assay using a spectrophotometer (Hitachi U3000) at $595 \mathrm{~nm}$ with BSA (Sigma-Aldrich; Merck KGaA) as the standard. For immunoblotting, extracted proteins $(20 \mu \mathrm{g} /$ lane $)$ were separated by $8-10 \%$ SDS-PAGE and electrophoretically transferred to nitrocellulose membranes (Bio-Rad Laboratories, Inc.). After blocking with $5 \%$ non-fat dry milk for $1 \mathrm{~h}$ at $4{ }^{\circ} \mathrm{C}$, antibodies against p-mTOR (Ser2481; 1:500; cat. no. 09343; EMD Millipore), p-AKT (Ser473; 1:500; cat. no. sc-7985-R), p-AKT (Thr308; 1:1,000; cat. no. sc-135650), p-S6RP (1:500; cat. no. sc-293144), vascular cell adhesion molecule 1 (VCAM-1; 1:250; cat. no. sc-13160), ICAM-1 (1:500; cat. no. sc-8439), integrin $\beta 1$ (1:1,000; cat. no. sc-8978; all Santa Cruz Biotechnology, Inc.), human inducible factor- $1 \alpha$ (HIF-1 $\alpha$; 1:500; cat. no. NB100-105), VEGF (1:500; cat. no. NB100-664; both Novus Biologicals, LLC) or $\beta$-actin (1:5,000; cat. no. MAB1501; EMD Millipore) were diluted in PBS with $2.5 \%$ BSA and incubated with the membranes overnight at $4^{\circ} \mathrm{C}$ with gentle agitation. The membranes were subsequently incubated with diluted HRP-conjugated secondary antibodies (1:5,000; cat. nos. sc-2004 or sc-2005; Santa Cruz Biotechnology, Inc.) for a further $1 \mathrm{~h}$ at $4^{\circ} \mathrm{C}$. An Immobilon Western Chemiluminescent HRP Substrate (EMD Millipore) and a chemiluminescence imaging analyzer (GE ImageQuant TL 8.1; GE Healthcare Life Sciences) were used to detect the antigen-antibody complexes. Finally, a densitometric apparatus (Appraise densitometer; Beckman Coulter, Inc.) was used to quantify the density of the blot.

Statistical analysis. The statistical significance among experimental groups was evaluated using GraphPad Prism 5 software (GraphPad Software, Inc.) by one-way ANOVA followed by Tukey's multiple comparisons test. All data are presented at the mean \pm SEM. Three independent experiments were performed. $\mathrm{P}<0.05$ was considered to indicate a statistically significant difference.

\section{Results}

Effects of B19V-VPlu antibodies on HUVEC survival. To detect the toxic effects of the antibodies used in this study on HUVECs, the Trypan blue exclusion method was performed. The survival of HUVECs was unchanged by the presence of 10\% FCS, NH IgG, aPL IgG, B19V-VP1u IgG and B19V-NS1 IgG (Fig. 1). Although the survival of HUVECs in the presence of B19V-NS1 IgG was slightly lower compared with those in the presence of $10 \%$ FCS or the other antibodies, no statistical significance was detected among these treatments (Fig. 1). 

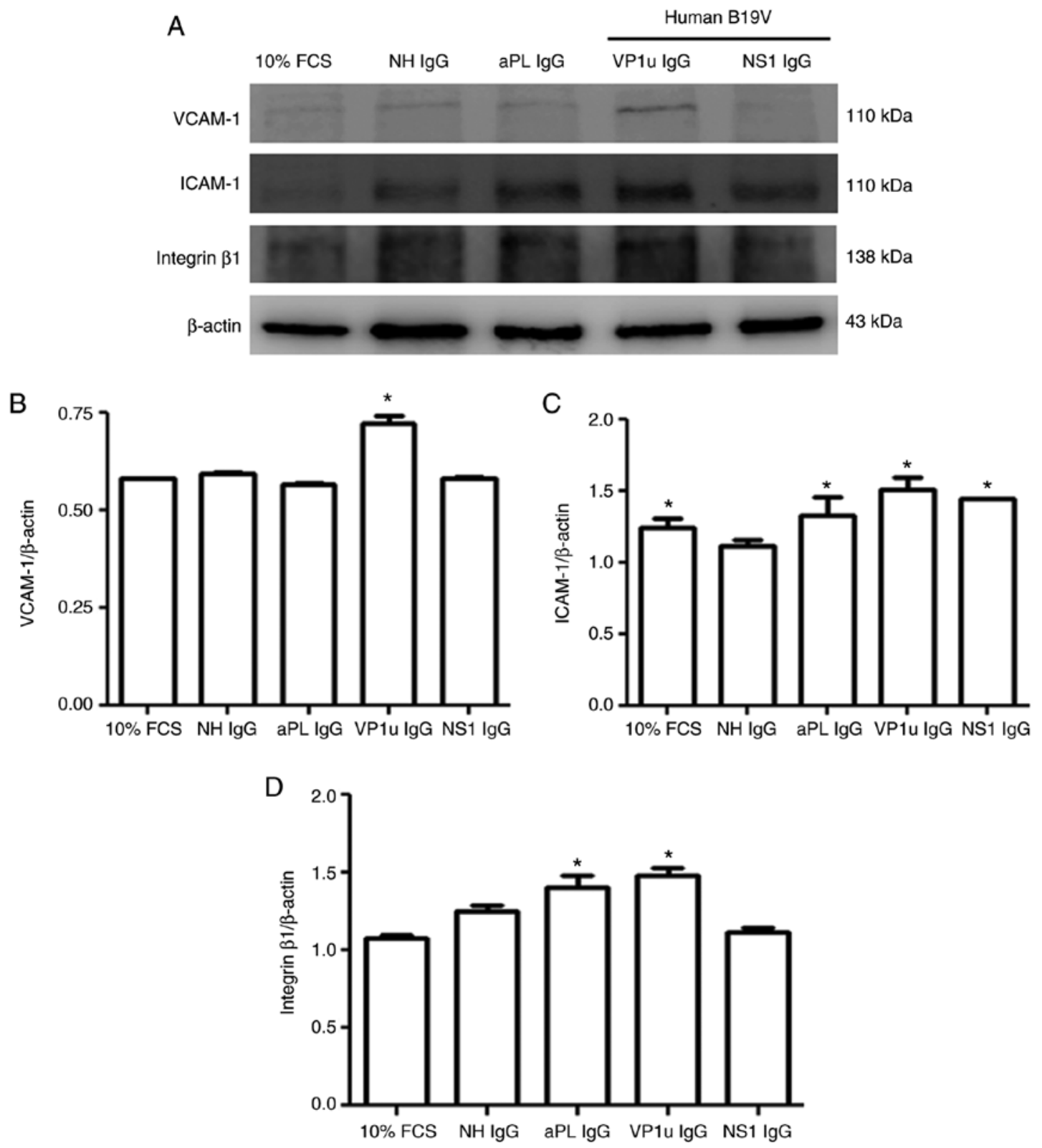

Figure 2. Expression of VCAM-1, ICAM-1 and integrin $\beta 1$. (A) Protein expression levels of VCAM-1, ICAM-1 and integrin $\beta 1$ in human umbilical vein endothelial cells treated with different antibodies were detected by immunoblotting. (B-D) Relative levels of (B) VCAM-1, (C) ICAM-1 and (D) integrin $\beta 1$ are based on $\beta$-actin expression. Similar results were observed in triplicate experiments. " $\mathrm{P}<0.05$ vs. cells treated with NH IgG. VCAM- 1 , vascular cell adhesion molecule 1; ICAM-1, intracellular adhesion molecule 1; NH, normal human; IgG, immunoglobulin G; FCS, fetal calf serum; aPL, anti-phospholipid antibodies; B19V, parvovirus B19; VP1u, VP1 unique region.

Effects of B19V-VPlu antibodies on adhesion molecules. To detect the effects of B19V-VPlu antibodies on adhesion molecules, the protein expression levels of VCAM-1, ICAM-1 and integrin $\beta 1$ were measured by immunoblotting. No significant difference in VCAM-1 level was observed among the HUVECs treated with $10 \%$ FCS, NH IgG, aPL IgG or B19V-NS1 $\mathrm{IgG}$, whereas significantly greater VCAM-1 expression was detected in the HUVECs that were treated with B19V-VP1u IgG compared with those treated with $\mathrm{NH} \mathrm{IgG} \mathrm{(Fig.} \mathrm{2A}$ and B). Significantly higher ICAM-1 expression was detected in the HUVECs treated with $10 \%$ FCS, aPL IgG, B19V-VP1u IgG or B19V-NS1 IgG compared with those treated with $\mathrm{NH}$ IgG (Fig. 2C). Significantly higher integrin $\beta 1$ expression was detected in the HUVECs treated with aPL IgG or B19V-VP1u
IgG compared with those treated with NH IgG (Fig. 2D). No difference in integrin $\beta 1$ expression was observed among the HUVECs treated with $10 \%$ FCS, NH IgG or B19V-NS1 IgG (Fig. 2D).

Effects of B19-VP1u antibodies on AKT-mTOR-S6RP signaling. To detect the effects of $\mathrm{B} 19 \mathrm{~V}$ antibodies on angiogenic signaling, the expression levels of proteins associated with the AKT-mTOR-S6RP pathway were detected by immunoblotting. Significantly higher protein expression levels of p-mTOR, p-AKT (Ser473), p-AKT (Thr308) and p-S6RP were detected in the HUVECs treated with $10 \%$ FCS compared with those treated with NH IgG (Fig. 3A). Significantly higher protein expression levels of p-mTOR, 
A
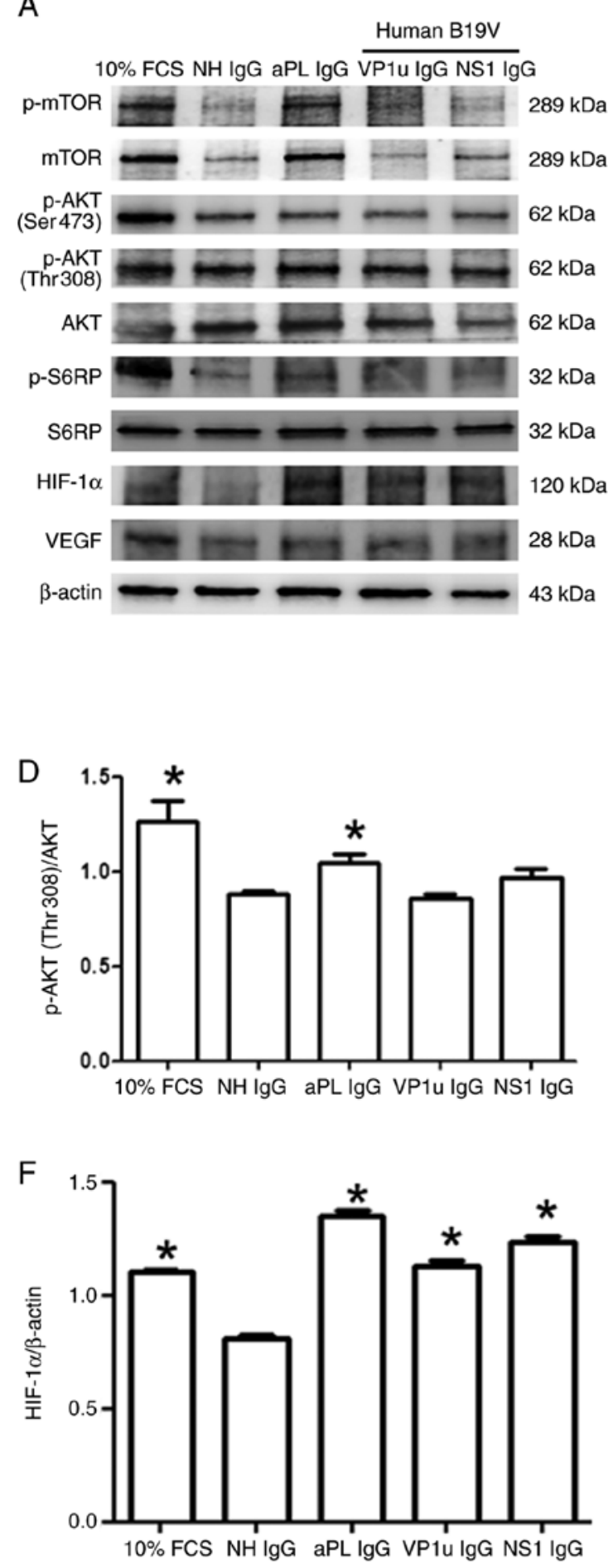
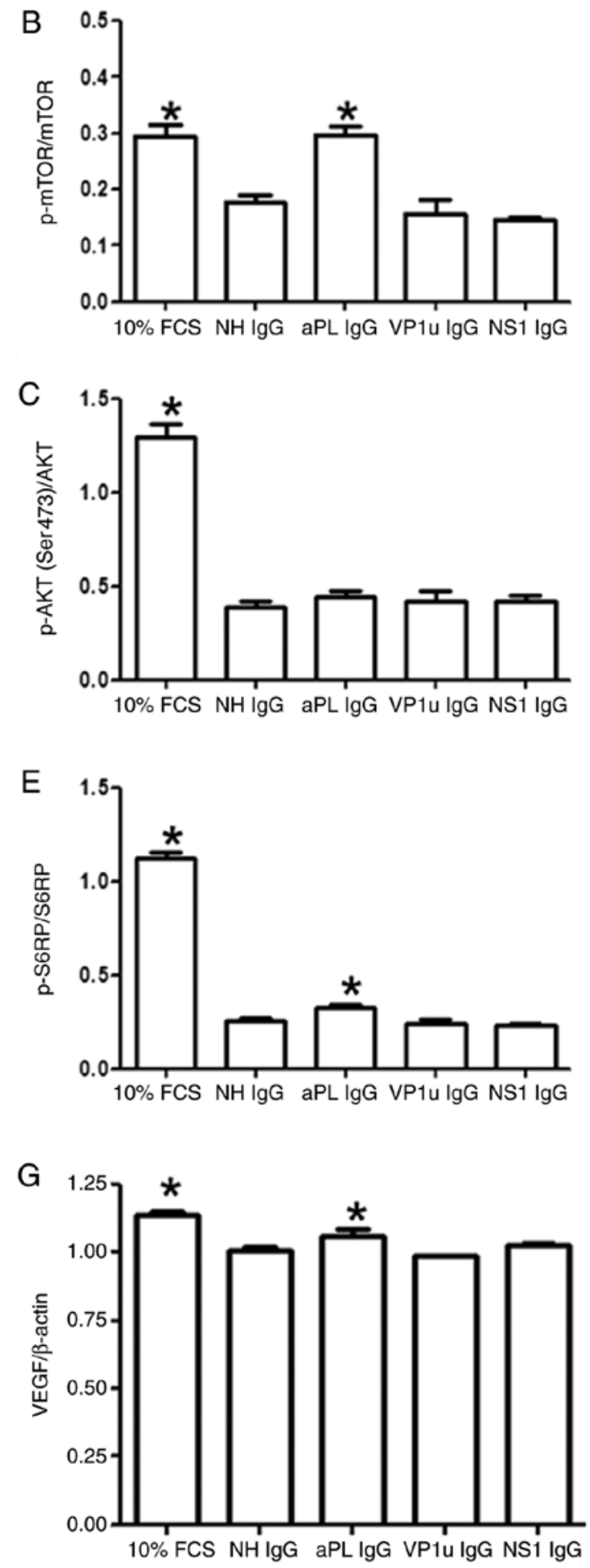

Figure 3. Expression and phosphorylation of mTOR, AKT, S6RP, HIF-1 $\alpha$ and VEGF proteins. (A) Protein expression levels in human umbilical vein endothelial cells treated with different antibodies were detected by immunoblotting: p-mTOR; mTOR; p-AKT (Ser473); p-AKT (Thr308); AKT; p-S6RP; S6RP; HIF-1 $\alpha$; and VEGF. (B-G) Relative levels of (B) p-mTOR (C) p-AKT (Ser473) (D) p-AKT (Thr308) (E) p-S6RP (F) HIF-1 $\alpha$ and (G) VEGF are based on its total protein expression or $\beta$-actin expression. Similar results were observed in triplicate experiments. " $\mathrm{P}<0.05$ vs. cells treated with NH IgG. p-, phosphorylated; S6RP, S6 ribosomal protein; $\mathrm{NH}$, normal human; HIF-1 $\alpha$, human inducible factor-1 $\alpha$; VEGF, vascular endothelial growth factor; IgG, immunoglobulin G; FCS, fetal calf serum; aPL, anti-phospholipid antibodies; B19V; parvovirus B19, VP1u, VP1 unique region.

p-AKT (Thr308) and p-S6RP were detected in HUVECs treated with aPL IgG compared with those treated with $\mathrm{NH}$ IgG (Fig. 3B-E). p-mTOR, p-AKT (Ser473 and Thr308) and p-S6RP expression levels in the HUVECs treated with B19V-VP1u IgG or B19V-NS1 IgG did not differ significantly compared with those cells treated with NH IgG (Fig. 3B-E).
The protein expression levels of HIF-1 $\alpha$ and VEGF were also measured. Significantly higher protein expression levels of HIF-1 $\alpha$ and VEGF were detected in the HUVECs treated with $10 \%$ FCS or aPL IgG compared with those treated with NH IgG (Fig. 3F and G). Significantly higher expression of HIF-1 $\alpha$ was detected in the HUVECs treated with 

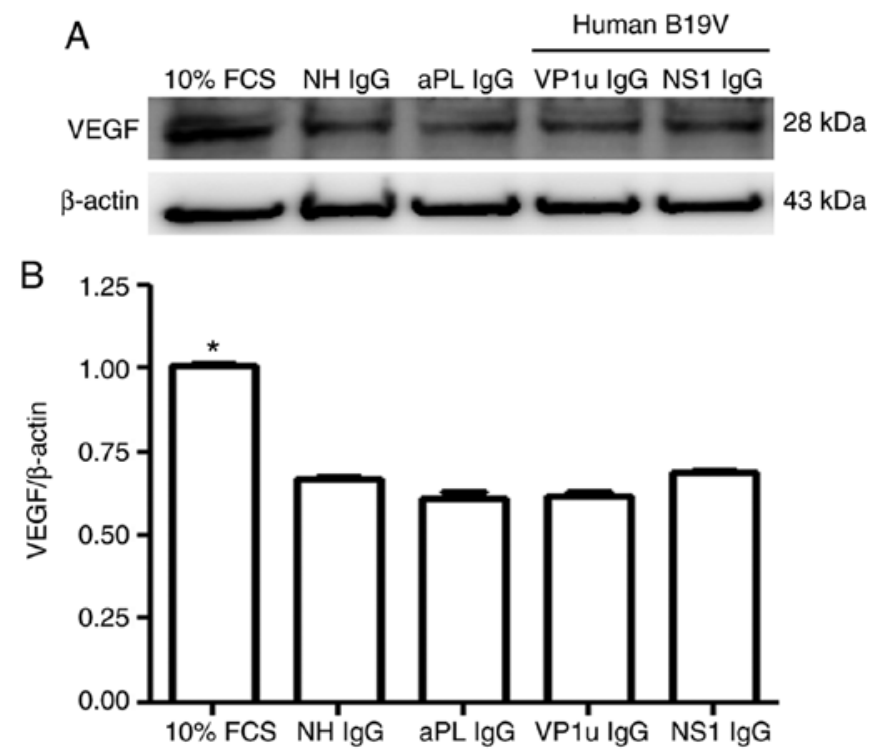

Figure 4. Expression of VEGF. (A) Protein expression level of VEGF in A549 cells treated with different antibodies was detected by immunoblotting. (B) Relative level of VEGF is based on $\beta$-actin expression. Similar result were observed in triplicate experiments. ${ }^{*} \mathrm{P}<0.05$ vs. cells treated with $\mathrm{NH}$ IgG. NH, normal human; VEGF, vascular endothelial growth factor; IgG, immunoglobulin G; FCS, fetal calf serum; aPL, anti-phospholipid antibodies; B19V, parvovirus B19; VP1u, VP1 unique region.

B19V-VP1u IgG or B19V-NS1 IgG compared with those treated with NH IgG (Fig. 3F). Notably, no significant difference in VEGF expression was observed among the HUVECs that were treated with $\mathrm{NH}$ IgG, B19V-VP1u IgG or B19V-NS1 IgG (Fig. 3G).

Effects of B19V-VPlu antibodies on VEGF expression in A549 cells. Since the A549 cell line is a well-known and popular model for studying angiogenesis in cancer $(23,24)$, the present study further investigated the effects of B19-VP1u antibodies on angiogenesis by detecting VEGF expression in A549 cells via immunoblotting. Significantly higher protein expression of VEGF was detected in A549 cells that were treated with $10 \%$ FCS compared with those treated with $\mathrm{NH} \operatorname{IgG}$, whereas no significant difference in VEGF protein expression was observed in the A549 cells treated with $\mathrm{NH} \operatorname{IgG}$, aPL IgG, B19V-VP1u IgG or B19V-NS1 IgG (Fig. 4).

\section{Discussion}

The engagement of $\beta 2 \mathrm{GPI}$ molecules at vascular endothelial cell membranes and antibodies against $\beta 2$ GPI can induce pro-coagulant and pro-inflammatory phenotypes, such as elevated expression levels of ICAM-1, VCAM-1, E-selectin and various pro-inflammatory cytokines $(18,26,27)$. Similar results have indicated that antibodies against B19-VP1u upregulate adhesion molecules, including ICAM-1, VCAM-1 and E-selectin in HUVECs by activating p-p38 MAPK signaling (17). Notably, some studies have indicated that antibodies against $32 \mathrm{GPI}$ phosphorylate AKT (Ser473) at the plasma membrane and activate PI3K-dependent AKT-mTOR signaling and lead to the development of vascular lesions and/or angiogenesis $(22,28,29)$. Although antibodies against B19V-VP1u have been reported to exhibit activity similar to that of aPL by inducing pro-inflammatory cytokines and adhesion molecules in APS $(8,17)$, little is known regarding the role of B19V-VP1u antibody in vascular intimal hyperplasia. In the present study, the induction of adhesion molecules, such as VCAM- 1 , ICAM- 1 and integrin $\beta 1$, but not AKT-mTOR signaling, was observed in HUVECs that had been treated with B19V-VPlu antibody. These findings are the first to reveal the role of antibodies against B19V-VP1u in activating adhesion molecules but not angiogenic signaling. However, the precise mechanism by which B19V-VPlu antibodies induce adhesion molecules requires further investigation.

The role of aPLs in angiogenesis is controversial. A previous study reported that aPLs in serum from patients with APS reduce in vitro angiogenesis to a level below that induced by serum from normal individuals (30). An in vivo experiment in which angiogenesis was impaired in aPL-inoculated mice revealed a similar result (20). In contrast, the induction of mTOR signaling, a key pathway of angiogenesis (31), has been observed in an in vitro study (22). These findings have attracted attention and motivated discussion of the role of aPLs in angiogenesis. Notably, the aPLs that were obtained from patients with APS in a previous study were a mixture of anti-32GPI, cardiolipin and anti-lupus anticoagulant antibodies (22). Since the role of each antibody in aPLs in angiogenesis is unclear, further investigations are required to identify the actual role of each type of antibody in aPLs in angiogenic signaling.

As well as being associated with autoimmune disorders, B19V has been associated with a variety of cancer types (32). A systematic review and meta-analysis of the literature revealed an association between B19V infection, and both testicular cancer and leukemia $(33,34)$. Nested PCR has revealed that B19V DNA and VP1/VP2 protein are more prevalent in tissues of colon adenocarcinomas compared with in adjacent noncancerous tissues, polyps and normal controls (35). A previous study reported significantly higher B19V capsid protein levels in thyroid tissues from patients with papillary thyroid carcinoma (PTC) than in the non-neoplastic adjacent tissues and in thyroid tissues from healthy controls, suggesting a possible role of B19V in the pathogenesis of PTC (36). These findings imply the possible involvement of B19V in cancer development. Since antibodies against B19V-VP1u exhibit similar activity to that of $\beta 2 \mathrm{GPI}$ antibodies, the present study investigated the effects of antibodies against B19V-VP1u on angiogenic signaling, which is an important indicator of tumorigenesis. Notably, antibodies against B19V-VP1u had no effect on the induction of mTOR angiogenic signaling and VEGF expression, which suggests that B19V-VPlu antibodies may have no effect on angiogenesis in cancer.

In summary, the present study, to the best of our knowledge, is the first to report that B19V-VPlu antibodies induce the expression of adhesion molecules, but not AKT-mTOR signaling and VEGF expression. Therefore, this implies a differential role of B19V-VPlu antibodies compared with aPLs on angiogenesis in APS.

\section{Acknowledgements}

Not applicable. 


\section{Funding}

This study was supported by the Chung Shan Medical University and Chi-Mei Medical Center Cooperative Project (grant nos. CSMU-CMMC-107-01 and CMCSMU10701). Consumptive materials were partially supported by the Chunghua Christian Hospital (grant no. 104-CCH-IRP-018) and Ministry of Science and Technology (grant no. MOST-10 6-2314-B040-023), Taiwan. The funders had no role in study design, data collection and analysis, decision to publish, or preparation of the manuscript.

\section{Availability of data and materials}

All data generated or analyzed during this study are included in this published article.

\section{Authors' contributions}

CCS, CCC, TCH and BST conceived and designed the experiments and analyzed the data; $\mathrm{CHH}$ and $\mathrm{TCH}$ performed the experiments and analyzed the data; TCH and BST drafted and revised the manuscript. All authors read and approved the final manuscript.

\section{Ethics approval and consent to participate}

Not applicable.

\section{Patient consent for publication}

Not applicable.

\section{Competing interests}

The authors declare that they have no competing interests.

\section{References}

1. Anderson MJ, Jones SE, Fisher-Hoch SP, Lewis E, Hall SM, Bartlett CL, Cohen BJ, Mortimer PP and Pereira MS: Human parvovirus, the cause of erythema infectiosum (fifth disease)? Lancet 1: 1378, 1983.

2. Reid DM, Reid TM, Brown T, Rennie JA and Eastmond CJ: Human parvovirus-associated arthritis: A clinical and laboratory description. Lancet 1: 422-425, 1985.

3. Serjeant GR, Topley JM, Mason K, Serjeant BE, Pattison JR, Jones SE and Mohamed R: Outbreak of aplastic crises in sickle cell anaemia associated with parvovirus-like agent. Lancet 2: 595-597, 1981.

4. Brown T, Anand A, Ritchie LD, Clewley JP and Reid TM: Intrauterine parvovirus infection associated with hydrops fetalis. Lancet 2: 1033-1034, 1984.

5. Meyer O: Parvovirus B19 and autoimmune diseases. Joint Bone Spine 70: 6-11, 2003.

6. Kerr JR: The role of parvovirus B19 in the pathogenesis of autoimmunity and autoimmune disease. J Clin Pathol 69: 279-91, 2016.

7. Dorsch S, Liebisch G, Kaufmann B, von Landenberg P, Hoffmann JH, Drobnik W and Modrow S: The VP1 unique region of parvovirus B19V and its constituent phospholipase A2-like activity. J Virol 76: 2014-2018, 2002.

8. Tzang BS, Lee YJ, Yang TP, Tsay GJ, Shi JY, Tsai CC and Hsu TC Induction of antiphospholipid antibodies and antiphospholipid syndrome-like autoimmunity in naive mice with antibody against human parvovirus B19 VP1 unique region protein. Clin Chim Acta 382: 31-36, 2007.
9. Tzang BS, Tsay GJ, Lee YJ, Li C, Sun YS and Hsu TC: The association of VP1 unique region protein in acute parvovirus B19 infection and anti-phospholipid antibody production. Clin Chim Acta 378: 59-65, 2007

10. Lin CY, Chiu CC, Cheng J, Lin CY, Shi YF, Tsai CC, Tzang BS and Hsu TC: Antigenicity analysis of human parvovirus B19-VP1u protein in the induction of anti-phospholipid syndrome. Virulence 9: 208-216, 2018.

11. Von Landenberg P, Lehmann HW, Knöll A, Dorsch S and Modrow S: Antiphospholipid antibodies in pediatric and adult patients with rheumatic disease are associated with parvovirus B19 infection. Arthritis Rheum 48: 1939-1947, 2003.

12. Lunardi C, Tinazzi E, Bason C, Dolcino M, Corrocher R and Puccetti A: Human parvovirus B19 infection and autoimmunity. Autoimmun Rev 8: 116-120, 2008.

13. Miyakis S, Lockshin MD, Atsumi T, Branch DW, Brey RL, Cervera R, Derksen RH, DE Groot PG, Koike T, Meroni PL, et al: International consensus statement on an update of the classifi cation criteria for definite antiphospholipid syndrome (APS). J Thromb Haemost 4: 295-306, 2006.

14. Linnemann B: Antiphospholipid syndrome - an update. Vasa 47: 451-464, 2018.

15. Chen DY, Tzang BS, Chen YM, Lan JL, Tsai CC and Hsu TC: The association of anti-parvovirus B19-VP1 unique region antibodies with antiphospholipid antibodies in patients with antiphospholipid syndrome. Clin Chim Acta 411: 1084-1089, 2010.

16. Loizou S, Cazabon JK, Walport MJ, Tait D and So AK: Similarities of specificity and cofactor dependence in serum antiphospholipid antibodies from patients with human parvovirus B19 infection and from those with systemic lupus erythematosus. Arthritis Rheum 40: 103-108, 1997.

17. Tzang BS, Tsai CC, Chiu CC, Shi JY and Hsu TC: Up-regulation of adhesion molecule expression and induction of TNF-alpha on vascular endothelial cells by antibody against human parvovirus B19 VP1 unique region protein. Clin Chim Acta 395: 77-83, 2008.

18. George J, Blank M,Levy Y, Meroni P, Damianovich M, Tincani A and Shoenfeld Y: Differential effects of anti-beta2-glycoprotein I antibodies on endothelial cells and on the manifestations of experimental antiphospholipid syndrome. Circulation 97: 900-906, 1998

19. Vega-Ostertag M, Casper K, Swerlick R, Ferrara D, Harris EN and Pierangeli SS: Involvement of p38 MAPK in the up-regulation of tissue factor on endothelial cells by antiphospholipid antibodies. Arthritis Rheum 52: 1545-1554, 2005.

20. Di Simone N, Di Nicuolo F, D'Ippolito S, Castellani R, Tersigni C, Caruso A, Meroni P and Marana R: Antiphospholipid antibodies affect human endometrial angiogenesis. Biol Reprod 83: 212-219, 2010.

21. Velásquez M, Rojas M, Abrahams VM, Escudero $\mathrm{C}$ and Cadavid ÁP: Mechanisms of endothelial dysfunction in antiphospholipid syndrome: Association with clinical manifestations. Front Physiol 9: 1840, 2018.

22. Canaud G, Bienaimé F, Tabarin F, Bataillon G, Seilhean D, Noël LH, Dragon-Durey MA, Snanoudj R, Friedlander G, Halbwachs-Mecarelli L, et al: Inhibition of the mTORC pathway in the antiphospholipid syndrome. N Engl J Med 371: 303-312, 2014.

23. Sakamoto Y, Terashita N, Muraguchi T, Fukusato T and Kubota S: Effects of epigallocatechin-3-gallate (EGCG) on A549 lung cancer tumor growth and angiogenesis. Biosci Biotechnol Biochem 77: 1799-1803, 2013.

24. Amann A, Zwierzina M, Koeck S, Gamerith G, Pechriggl E, Huber JM, Lorenz E, Kelm JM, Hilbe W, Zwierzina H and Kern J: Development of a 3D angiogenesis model to study tumour-endothelial cell interactions and the effects of anti-angiogenic drugs. Sci Rep 7: 2963, 2017.

25. Tsai CC, Chiu CC, Hsu JD, Hsu HS, Tzang BS and Hsu TC: Human parvovirus B19 NS1 protein aggravates liver injury in NZB/W F1 mice. PLoS One 8: e597242013, 2013.

26. Raschi E, Testoni C, Borghi MO, Fineschi S and Meroni PL: Endothelium activation in the anti-phospholipid syndrome. Biomed Pharmacother 57: 282-286, 2003.

27. Meroni PL, Raschi E, Testoni C and Borghi MO: Endothelial cell activation by antiphospholipid antibodies. Clin Immunol 112: 169-174, 2004.

28. Shi T, Giannakopoulos B, Yan X, Yu P, Berndt MC, Andrews RK, Rivera J, Iverson GM, Cockerill KA, Linnik MD and Krilis SA: Anti-beta2-glycoprotein I antibodies in complex with beta2-glycoprotein I can activate platelets in a dysregulated manner via glycoprotein Ib-IX-V. Arthritis Rheum 54: 2558-2567, 2006. 
29. de Laat B, Derksen RH, van Lummel M, Pennings MT and de Groot PG: Pathogenic anti-beta2-glycoprotein I antibodies recognize domain I of beta2-glycoprotein I only after a conformational change. Blood 107: 1916-1924, 2006.

30. D'Ippolito S, Marana R, Di Nicuolo F, Castellani R, Veglia M, Stinson J, Scambia G and Di Simone N: Effect of low molecular weight heparins (LMWHs) on antiphospholipid antibodies (aPL)-mediated inhibition of endometrial angiogenesis. PLoS One 7: e29660, 2012

31. Mitsiades CS, Mitsiades N and Koutsilieris M: The Akt pathway: Molecular targets for anti-cancer drug development. Curr Cancer Drug Targets 4: 235-256, 2004.

32. Jitschin R, Peters O, Plentz A, Turowski P, Segerer H and Modrow S: Impact of parvovirus B19 infection on paediatric patients with haematological and/or oncological disorders. Clin Microbiol Infect 17: 1336-1342, 2011.
33. Yousif L, Hammer GP, Blettner M and Zeeb H: A systematic literature review and meta-analysis. J Med Virol 85: 2165-2175, 2013.

34. Ibrahem WN, Hasony HJ and Hassan JG: Human parvovirus B19 in childhood acute lymphoblastic leukaemia in Basrah. J Pak Med Assoc 64: 9-12, 2014.

35. Li Y, Wang J, Zhu G, Zhang X, Zhai H, Zhang W, Wang W and Huang G: Detection of parvovirus B19 nucleic acids and expression of viral VP1/VP2 antigen in human colon carcinoma. Am J Gastroenterol 102: 1489-1498, 2007.

36. Wang JH, Zhang WP, Liu HX, Wang D, Li YF, Wang WQ, Wang L, He FR, Wang Z, Yan QG, et al: Detection of human parvovirus B19 in papillary thyroid carcinoma. Br J Cancer 98: 611-618, 2008. 